\title{
Targeting Spindle-Associated Protein HAUS6 Prevents Cell Growth of Colorectal Cancer Cells by Activating the p53/p21 Pathway
}

\section{Aling Shen}

Fujian University of Traditional Chinese Medicine

Liya Liu

Fujian University of Traditional Chinese Medicine

\section{Yue Huang}

Fujian University of Traditional Chinese Medicine

\section{Zhiqing Shen}

Fujian University of Traditional Chinese Medicine

\section{Meizhu Wu}

Fujian University of Traditional Chinese Medicine

\section{Xiaoping Chen}

Fujian University of Traditional Chinese Medicine

\section{Xiangyan Wu}

Fujian University of Traditional Chinese Medicine

Xiaoying Lin

Fujian University of Traditional Chinese Medicine

\section{Youqin Chen}

Case Western Reserve University

\section{Li Li}

Fujian Provincial Hospital

\section{Ying Cheng}

Fujian University of Traditional Chinese Medicine Jianfeng Chu

Fujian University of Traditional Chinese Medicine

\section{Thomas Sferra}

Case Western Reserve University

\section{Lihui Wei}

Fujian University of Traditional Chinese Medicine

\section{Qunchuan Zhuang}

Fujian University of Traditional Chinese Medicine Jun Peng ( $\nabla$ pjunlab@hotmail.com ) 
Fujian University of Traditional Chinese Medicine

\section{Research}

Keywords: HAUS6, tumor growth, proliferation, p53/p21 pathway, survival

Posted Date: September 23rd, 2020

DOl: https://doi.org/10.21203/rs.3.rs-76669/v1

License: (c) (1) This work is licensed under a Creative Commons Attribution 4.0 International License. Read Full License 


\section{Abstract}

Background: HAUS6 participates in microtubule-dependent microtubule amplification, but its role in malignancies including colorectal cancer (CRC) has not been explored. We therefore assessed the potential oncogenic activities of HAUS6 in CRC.

Methods: We investigated HAUS6 expression and its prognostic value in CRC by microarray, analysis of public datasets, quantitative reverse transcription - polymerase chain reaction (qRT-PCR) and immunohistochemistry. Biological functions of HAUS6 were investigated using loss-of-function and gainof-function assays in vivo and/or in vitro. Effectors downstream of HAUS6 were identified using cDNA microarray, bioinformatics analysis, qRT-PCR and western-blotting in CRC cells. The putative mechanism by which HAUS6 knockdown elevated p53 and p21 expression were assessed by rescue experiment. The combination effects of HAUS6 knockdown and 5-fluorouracil (5-FU) treatment in cultured CRC cells were further evaluated.

Results: HAUS6 mRNA and protein expression is higher in CRC tissues, and high HAUS6 expression is correlated with shorter overall survival in CRC patients. HAUS6 knockdown in CRC cell lines suppressed cell growth in vitro and in vivo by inhibiting cell viability, survival and arresting cell cycle progression at G0/G1, while HAUS6 over-expression increased cell viability. We showed that these effects are dependent on activation of the p53/p21 signalling pathway by reducing p53 and p21 degradation. Moreover, HAUS6 knockdown enhanced the effects of 5-FU treatment in CRC cells by increasing activation of the p53/p21 pathway.

Conclusions: Our study highlights a potential oncogenic role for HAUS6 in CRC. Targeting HAUS6 may be a promising novel prognostic marker and chemotherapeutic target for treating CRC patients.

\section{Background}

Colorectal cancer (CRC) is one of the leading causes of mortality worldwide [1]. Progression from microscopic pre-neoplastic lesions to larger adenomas involves a cascade of mechanisms including tumor suppressor gene mutation and bypass of cell cycle checkpoints [2]. Unfortunately, current treatment strategies have low success rates due to our poor understanding of the molecular mechanisms behind CRC progression. Hence, there is an urgent demand to understand the fundamental mechanisms underlying CRC development and to find novel molecular targets [3].

Follow-up analysis of gene expression profile microarrays from our previous study (GEO Submission: GSE113513) [4] showed that levels of mRNA encoding HAUS augmin like complex subunit 6 (HAUS6) are higher in CRC tissues than in adjacent noncancerous colorectal tissues. Our preliminary experiments further suggested that HAUS6 knockdown inhibits growth of CRC cells in culture (data not shown). These results suggest that HAUS6 has oncogenic potential in CRC. 
HAUS6 is one of the 8 subunits of the augmin complex [5, 6], which is required for microtubule (MT)dependent MT amplification during cell division independently of the centrosomes and chromatin [7-10]. HAUS6 promotes MT-dependent MT amplification by interacting with and recruiting neural precursor cellexpressed developmentally down-regulated protein 1 (NEDD1), a subunit of the NEDD1- $y$-tubulin ring complex. Depletion of HAUS6 reduces MT density in the bipolar spindle [6]. Dissociation of the augmin

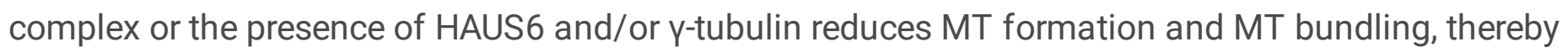
affecting spindle assembly during mitosis and MT organization in interphase [11, 12]. MTs, spindle poles and chromosomes form the main elements of the spindle architecture [13, 14], which plays an essential role in organizing bipolar spindles and chromosomal segregation during mitosis [15]. Failure of these processes results in mitotic arrest and apoptosis and contributes to chromosome mis-segregation and aneuploidy, a hallmark of cancer [16]. However, the role of HAUS6 in cancer has never been evaluated to our knowledge. In the present study, we investigated the effects of HAUS6 expression on patient prognosis and on viability and proliferation of CRC cell lines in vitro and in vivo, as well as its potential underlying mechanisms.

\section{Materials And Methods}

\section{Bioinformatic analysis}

HAUS6 expression in CRC and noncancerous colorectal tissues was compared using the Oncomine database (www.oncomine.org), as described previously [4]. Correlation of HAUS6 expression with CRC patient survival was performed using the R2 Bioinformatic Platform (http://r2.amc.nl), as described previously [4]. The threshold of significance was set at $p<0.05$.

\section{Patients and specimens}

Both CRC and matched adjacent non-cancerous tissues were collected from $36 \mathrm{CRC}$ patients admitted to the First People's Hospital Affiliated with Fujian University of Traditional Chinese Medicine (FJTCM). Clinicopathologic characteristics of patients are summarized in Supplementary Table S2. No patients received radio- or chemotherapy prior to surgery. Tissues were snap-frozen and stored in liquid nitrogen for quantitative RT-PCR or processed for immunohistochemistry. Samples were stained with hematoxylin and eosin, then examined by experienced pathologists, who assigned pathologic tumor-node-metastasis ( $\mathrm{p}$-TNM) grades according to International Union Against Cancer guidelines.

In addition, cDNA from 80 primary CRC and 15 adjacent noncancerous colorectal tissues were obtained from the cDNA-HColA095Su01 tissue cDNA array (Shanghai Outdo Biotech, Supplementary Table S3). A further 280 primary CRC and 260 adjacent noncancerous colorectal tissues were obtained from the tissue microarray (TMA) (Shanghai Outdo Biotech; Supplementary Table S4).

\section{Quantitative RT-PCR}

Cells and tissue samples were lysed with RNAiso Plus reagent and total RNA was extracted according to the manufacturer's instructions. A total of $1 \mu \mathrm{g}$ RNA was reverse-transcribed into cDNA using PrimeScript 
RT reagent kit and RT-qPCR was performed using SYBR Premix Ex Taq and the ABI 7500 Fast Real-Time PCR System (Applied Biosystems) according to manufacturer protocols. Primer sequences against HAUS6, TP53, CDKN1A and GAPDH are shown in Supplementary Table S5. GAPDH was used as an internal control.

\section{Immunohistochemical staining}

Tissues were processed using routine immunohistochemistry methods as in our previous study [4]. Tissue specimens collected in our laboratory were fixed, sectioned and stained with primary antibodies against HAUS6 and Ki-67 then secondary biotinylated antibody (see supplementary Table S6), followed by incubation with horseradish peroxidase-conjugated streptavidin. Background staining was assessed by omitting the primary antibody. Five randomly selected fields were examined using a Leica microscope at $40 \times$ magnification.

For tissue arrays, slides were incubated with primary antibody against HAUS6 (dilution 1:500, Genetex) as described $[4,17]$. Images were captured using a Nano Zoomer $2.0 \mathrm{HT}$ slide scanner and processed using Nano Zoomer Digital Pathology View 1.6 software. Staining intensity and the percentage of positively stained cells were determined as described $[4,18]$. Protein expression was calculated by multiplying the intensity and percentage scores together, giving expression scores ranging from 0 to 12 .

\section{Cell culture}

Human CRC cell lines HCT116 and RKO were purchased from the Cell Bank of the Chinese Academy of Sciences (Shanghai, China). Wild-type HCT116 cells (HCT116/p53 ${ }^{+/+}$) and p53 knockout HCT116 cells (HCT116/p53 ${ }^{-/-}$) were a gift from Dr. Yao Lin (Fujian Normal University, Fujian, China) and originally obtained from Dr Bert Vogelstein (Johns Hopkins University, Baltimore, MD). All cells were cultured in RPMI-1640 media supplemented with $10 \%$ fetal bovine serum, as well as $100 \mathrm{units} / \mathrm{mL}$ penicillin and $100 \mu \mathrm{g} / \mathrm{mL}$ streptomycin at $37^{\circ} \mathrm{C}$ in a humidified atmosphere of $5 \% \mathrm{CO}_{2}$. Cells were verified using short tandem repeat genotyping and examined for mycoplasma contamination using RT-PCR.

5-FU (Sigma-Aldrich) was dissolved in DMSO and used to treat control and HAUS6 knockdown cells; DMSO was used as a vehicle control.

\section{Lentiviral transduction}

Lentiviruses encoding shRNA against HAUS6 or CDKN1A or encoding control shRNA (Supplementary Table S7 for sequences) and enhanced green fluorescent protein (EGFP) under control of the CMV promoter were obtained from Shanghai GeneChem (Shanghai, China). CRC cells were transduced with lentivirus at a multiplicity of infection (MOI) of 10 for $72 \mathrm{~h}$ before analysis. To examine effects of HAUS6 over-expression on cell proliferation, HCT116 cells were transduced for $72 \mathrm{~h}$ with a lentiviral vector $(\mathrm{MOI}=$ 10) encoding full-length human HAUS6 (2,946 nt, GenBank accession NM_017645; Shanghai GeneChem). Transductants were selected for 2 weeks using $1 \mu \mathrm{g} / \mathrm{mL}$ puromycin.

\section{Cell viability analysis}


Cell viability was measured every day for 5 days by CCK-8 assay. Briefly, transduced cells were reseeded into 96-well plates at a density of 2000 cells/well with $100 \mu \mathrm{L}$ medium/well. CCK-8 dye was added to each well and cells were incubated for $2 \mathrm{~h}$ at $37^{\circ} \mathrm{C}$. Cell viability was measured at $450 \mathrm{~nm}$.

\section{Cell survival analysis}

Transduced cells were reseeded into 12-well or 6 well plates at a density of 500 or 1000 cells/well and incubated for $10-12$ days at $37^{\circ} \mathrm{C}$ under $5 \% \mathrm{CO} 2$ in a humidified incubator. Colonies were fixed with $4 \%$ paraformaldehyde for 20 min and stained with $0.1 \%$ of crystal violet for $20 \mathrm{~min}$. Colonies were photographed and the number of colonies counted. Data were normalized relative to control cells.

\section{Cell cycle analysis}

Transduced cells were collected and fixed with cold $70 \%$ ethanol at $4{ }^{\circ} \mathrm{C}$ overnight. The cells were then washed three times with PBS and incubated with FxCycle PI/RNase Staining Solution for 30 min. Fluorescence-activated cell sorting was performed using fluorescence activated cell sorting (FACS) Caliber (Becton Dickinson), and ModfitLT version 3.0 (Verity Software House) was used to analyze the cell cycle based on DNA content in different phases.

\section{In vivo experiments}

Male pathogen-free athymic nude mice (6-8 weeks old) were purchased from Shanghai Laboratory Animal Co., Ltd. (Shanghai, China) and maintained in a specific pathogen-free (SPF) facility.

Tranduced CRC cells $\left(1 \times 10^{6}\right)$ in $100 \mu \mathrm{L}$ RPMI-1640 medium containing $50 \%$ matrigel were subcutaneously injected into the flank of nude mice $(n=8)$. Tumor growth was monitored from day 7 after injection on every second day for 19 days. Tumor volume was calculated as (1/2) (length $\times$ width $\left.^{2}\right)$, where length and width refer to the longest longitudinal and transverse diameters.

Tumor growth was also measured in terms of GFP intensity using an IVIS Spectrum whole live-animal imaging system (PerkinElmer). Mice were anesthetized with isoflurane and sacrificed by neck dislocation on day 26 after injection, and tumors were dissected and weighed. Collected tissues were processed for immunohistochemistry.

\section{Microarray analysis}

HCT116 cells were transduced with lentivirus encoding shRNA specific for HAUS6 or control shRNA for $72 \mathrm{~h}$. Briefly, total RNA was extracted with Trizol reagent and analyzed by CapitalBio using the human GeneChip Primeview array (Affymetrix) according to the manufacturer's instructions. Microarray images were scanned using GeneChip Scanner 3000 and analyzed using GeneChip GCOS 1.4 software (Affymetrix). Genes with a $p$-value $<0.05$ and fold change $>2$ were defined as differentially expressed. The raw microarray data can be found in the NCBI Gene Expression Omnibus (GEO Submission: GSE140326). Enriched pathways in DEGs were identified using KEGG pathway enrichment analysis.

\section{Western blot analysis}


Cells were lysed with RIPA lysis buffer and the protein concentration was determined using BCA assay. The proteins $(50 \mu \mathrm{g})$ were then separated by SDS-PAGE and transferred onto a nitrocellulose membrane. The membranes were blocked with BSA and incubated overnight at $4{ }^{\circ} \mathrm{C}$ with primary antibody against HAUS6, p53, or p21 (Supplementary Table 6). The membranes were then washed three times with TBST, and incubated at room temperature for $1 \mathrm{~h}$ with horseradish peroxidase-conjugated goat anti-rabbit. The blots were visualized using enhanced chemiluminescence according to the manufacturer's protocols.

\section{Statistical analysis}

Statistical analysis was performed using SPSS 20.0 (IBM, Chicago, IL, USA). Data are presented as mean \pm SD. The median HAUS6 expression in CRC tissues was used as the cutoff to divide tumors into "low expression" or "high expression". The relationship between HAUS6 expression and overall survival was analyzed using Kaplan-Meier curves and the log-rank test. Differences between two groups were assessed using the independent or paired Student's $t$ test, while differences among three or more groups were assessed using one-way ANOVA. Survival differences were assessed using the log-rank test. The correlation between HAUS6 and CDKN1A expression was analyzed using Pearson's correlation. A $p$-value of $<0.05$ was considered statistically significant.

\section{Study approval}

Collection of the clinic samples and related experiments were approved by the Ethics Committee of FJTCM. Written, informed consent was obtained from all patients or their families. All animal maintenance and procedures were performed in strict accordance with the "Guide for the Care and Use of Laboratory Animals" and the "Principles for the Utilization and Care of Vertebrate Animals" and approved by the Animal Committee of FJTCM.

\section{Results}

\section{HAUS6 is highly expressed in CRC tissues}

We first compared HAUS6 expression in cDNA samples from 14 matched pairs of primary CRC tumors and non-cancerous tissues from a cDNA array (GEO Submission: GSE113513). HAUS6 expression was higher in CRC tumors than non-cancerous tissues (Fig. 1A). Analysis of publicly available tumor expression data from the Oncomine database (http://www.oncomine.com/) also showed higher HAUS6 mRNA levels in CRC tissues than non-cancerous tissues (Supplementary Table S1). Quantitative RT-PCR analysis of HAUS6 expression in clinical samples of 36 CRC tissues and adjacent non-cancerous colorectal tissues from our laboratory and a CDNA tissue array containing 15 pairs of CRC primary tumors and surrounding non-cancerous tissues also showed higher HAUS6 mRNA expression in CRC tissues (Fig. 1B-C). Immunohistochemistry of 10 pairs of CRC samples randomly selected from the above mentioned 36 pairs, as well as analysis of a tissue microarray of 260 pairs of CRC samples (Shanghai Outdo Biotech), confirmed that HAUS6 protein levels were higher in CRC primary tumor tissues (Fig. 1DE). 


\section{HAUS6 expression is associated with poor patient prognosis}

Kaplan-Meier analysis of 80 patients whose primary tumor tissues were sampled on the cDNA array mentioned above did not reveal any difference in patient survival between tumors with low and high HAUS6 mRNA expression (data not shown). In contrast, analysis of data from 194 CRC patients (high expression: $n=79$; low expression: $n=115$ ) from the public microarray dataset using the R2 bioinformatic platform showed that high HAUS6 mRNA expression was associated with shorter overall survival (Fig. 2A). Similarly, tumors with high HAUS6 protein levels were associated with shorter overall survival in 280 CRC patients (Fig. 2B; high expression: $n=36$; low expression: $n=244$ ). Representative images of high or low expression of HAUS6 in CRC tissues are shown in Fig. 2C.

\section{HAUS6 knockdown inhibits CRC cell growth and proliferation in vitro}

We next assessed the effects of HAUS6 knockdown on growth and proliferation of CRC cell lines. Lentiviral transduction with vectors expressing short hairpin (sh)RNAs against HAUS6 down-regulated HAUS6, as expected (Fig. 3A-B; Supplementary Fig. S1A-B). CRC cells transduced with HAUS6 shRNAs had lower cell viability than those transduced with control RNAs (Fig. 3C; Supplementary Fig. S1C). In contrast, lentiviral transduction with vectors over-expressing HAUS6 up-regulated HAUS6 protein expression, and increased the cell viability of HCT116 cells based on the CCK8 assay (Fig. 3A-C, right panel). Moreover, HAUS6 knockdown also decreased the number of colonies formed by CRC cells (Fig. 3D). Cell cycle analysis revealed that HAUS6 knockdown increased the percentage of cells in G0/G1 and decreased the percentage of cells in S phase (Fig. 3E). However, HAUS6 knockdown did not affect cell apoptosis in HCT116 cells (Supplementary Fig. S2).

HAUS6 knockdown suppresses CRC cell growth and proliferation in vivo.

We next used a xenograft mouse model to assess the effects of HAUS6 knockdown on CRC cell growth and proliferation in vivo. HAUS6 knockdown strongly reduced tumor volume (Fig. 4A) and decreased fluorescence of tumor cells (Fig. 4B) in nude mice injected with GFP-expressing CRC cells. Final tumor weights were also lower in mice injected with HAUS6 knockdown cells than in those injected with control cells (Fig. 4C). Immunohistochemistry of the excised tumors showed that Ki-67 protein expression was decreased in HAUS6 knockdown cells (Fig. 4D), suggesting that HAUS6 knockdown suppresses CRC tumor growth by inhibiting cell proliferation.

HAUS6 knockdown suppresses cell proliferation by down-regulating p21.

Microarray analysis of HAUS6 knockdown HCT116 cells revealed a total of 274 differentially expressed genes (DEGs) in CRC (Fig. 5A-B; GEO Submission: GSE140326). CDKN1A (encoding p21 protein) was upregulated in HAUS6 knockdown cells (Fig. 5C), and this was confirmed in both HCT116 and RKO cells 
by quantitative RT-PCR (Fig. 5D) and western blotting (Fig. 5E). Transduction with shRNAs specific for HAUS6 and CDKN1A reversed the up-regulation of p21 observed in HAUS6 knockdown cells (Fig. 5F). HAUS6 knockdown decreased cell viability and colony formation, whereas CDKN1A knockdown had the opposite effect and attenuated the effects of HAUS6 knockdown (Fig. 5G-H). HAUS6 knockdown increased the percentage of cells in G0/G1 and reduced the percentage in S phase, which were reversed by CDKN1A knockdown (Fig. 5I). Analysis of microarray expression data (GEO Submission: GSE113513) showed that CDKN1A was down-regulated in CRC tissues (Fig. 5J) and its expression correlated negatively with HAUS6 expression (Fig. 5K).

\section{HAUS6 knockdown suppresses cell proliferation by activating the p53 pathway.}

KEGG pathway enrichment analysis of DEGs in HAUS6 knockdown cells identified the p53 pathway as the one of the most enriched signalling pathways (Fig. 6A). Given the key regulatory effects of $p 53$ on cell proliferation and CDKN1A transcription, we next explored the effect of HAUS6 on the p53 pathway. HAUS6 knockdown increased TP53 expression in HCT116 and RKO cells at the levels of mRNA (Fig. 6BC) and protein (Fig. 6D). The inhibitory effects of HAUS6 knockdown on cell viability were attenuated by knockout of p53 (Fig. 6E-F) or treatment with the p53 inhibitor PFT-a (Fig. 6G). Similarly, p53 knockout blocked the inhibitory effects of HAUS6 knockdown on cell survival (Fig. $6 \mathrm{H}$ ) and cell cycle progression (Fig. 6I-J). To determine the molecular mechanism by which HAUS6 knockdown regulates p53, we evaluated p53 protein stability in RKO cells expressing wild-type p53. Protein levels of p53 and its downstream p21 decreased over time in sh-Ctrl cells, but remained stable after HAUS6 knockdown (Fig. 6K).

\section{Targeting HAUS6 sensitizes CRC cells to 5-fluorouracil by activating the p53/p21 pathway.}

We further assessed the effect of HAUS6 on the response of CRC cells to 5-fluorouracil (5-FU) treatment. 5-FU treatment down-regulated HAUS6 and up-regulated p53 and p21 protein expression. These effects were further enhanced by HAUS6 knockdown (Fig. 7A). Moreover, HAUS6 knockdown reduced viability of HCT116 cells, regardless of whether they were treated with 5-FU (Fig. 7B). Combining HAUS6 knockdown with 5-FU led to greater suppression of HCT116 colony formation (Fig. 7C) than 5-FU alone, as well as higher percentages of cells in G0/G1 and G2/M and lower percentage in S phase (Fig. 7D-E).

\section{Discussion}

In this study, we show that the spindle-associated protein HAUS6 has an oncogenic role in CRC. HAUS6 mRNA and protein expression were both increased in CRC tissues compared to adjacent noncancerous colorectal tissues, and higher HAUS6 expression was correlated with shorter overall survival in CRC patients. HAUS6 knockdown suppressed tumor growth by inhibiting cell viability and survival as well as by arresting cells in G0/G1. HAUS6 knockdown exerted these effects by reducing p53 degradation and activating the $\mathrm{p} 53 / \mathrm{p} 21$ signaling pathway. 
Analysis from our own cDNA array [4] found that HAUS6 mRNA expression was increased in CRC tissues compared to adjacent noncancerous colorectal tissues. This was consistent with our analysis of multiple datasets from the Oncomine database, quantitative RT-PCR analysis of a cDNA tissue array, and immunohistochemical analysis of a tissue microarray. These results suggest that increased HAUS6 expression may be common in CRC and may play an essential role in CRC development. Moreover, survival analysis revealed a correlation between high HAUS6 mRNA and protein expression and shorter overall survival in CRC patients. This demonstrates that HAUS6 may be useful as a biomarker for CRC prognosis. However, HAUS6 expression should first be further investigated in other types of malignancies.

Cancer cells are characterized by an uncontrolled increase in cell proliferation [19, 20], which requires mitotic spindle formation. Nucleation of MTs is the initial step of mitotic spindle formation $[15,16]$, and loss-of-function in MT-dependent MT amplification results in mitotic arrest [21, 22]. Therefore, inhibition of mitotic spindle formation by targeting microtubule nucleation factors may be a promising anti-cancer strategy [23-25]. Many microtubule inhibitors, e.g. taxans, vinca alkaloids and paclitaxel, induce mitotic arrest by interfering with microtubule dynamics and have been used to treat cancer [26-28]. However, due to side effects in normal cells and acquired resistance in cancer cells, it is important to further explore the underlying mechanism of mitosis and find new targets for anticancer treatment. In the present study, we show that knockdown of the spindle assembly factor HAUS6 suppressed tumor growth in vivo and in vitro by inhibiting cell viability, survival and cell cycle progression. In contrast, HAUS6 over-expression obviously increased cell viability of HCT116 cells in vitro. We further show that HAUS6 knockdown enhanced the ability of 5-FU to decrease cell viability and survival and to arrest cells in G0/G1 and G2/M phases. These results suggest that HAUS6 may be a promising new target for anticancer treatments.

Microarray analysis of HAUS6 knockdown cells revealed 103 up-regulated genes and 171 down-regulated genes. Many of them, including CDKN1A [29], CyclinD1 [30], ROCK2 [31] and HMGA2 [32], have already been implicated in proliferation and cell cycle progression. One notable DEG was CDKN1A (p21), which arrests cell cycle progression at G1/S phase by binding to and inhibiting cyclin-dependent kinases [33]. We found that p21 protein levels were increased in HAUS6 knockdown cells, and that CDKN1A knockdown reversed the effects of HAUS6 knockdown on cell viability, cell survival and cell cycle arrest. Moreover, HAUS6 expression was inversely correlated with CDKN1A expression. These results indicate that CDKN1A may mediate the oncogenic effects of HAUS6. However, the regulatory effects of HAUS6 on p21 need to be further explored.

KEGG pathway enrichment analysis of DEGs in HAUS6 knockdown cells showed that the p53 pathway was one of the most enriched. The p53 protein upregulates p21 in response to stress stimuli by binding to two highly conserved p53 response elements in the p21 promoter [34, 35]. As a tumor suppressor, p53 is expressed at low levels under normal conditions due to MDM2, which mediates nuclear export of p53 and targets p53 for ubiquitination and degradation. Under stress conditions, p53 rapidly accumulates and activates p21 [33, 35, 36]. In our experiments, HAUS6 knockdown increased TP53 mRNA and protein expression. Knockout of p53 also abrogated the effects of HAUS6 knockdown on cell viability, survival and cell cycle arrest in HCT116 cells. Therefore, the suppression of tumor growth caused by HAUS6

Page 10/24 
knockdown depends at least partly on activation of p53. We further found that HAUS6 knockdown reduced the degradation of p53 and p21, suggesting a mechanism by which HAUS6 regulates p53.

Taken together, these results show that HAUS6 knockdown suppresses CRC tumor growth by increasing the stability of p53. Moreover, combining HAUS6 knockdown with 5-FU treatment led to even greater down-regulation of HAUS6 and up-regulation of p21 and p53 than 5-FU alone, suggesting a common therapeutic mechanism. The effect of HAUS6 on tumorigenesis and p53 expression is similar to that of Plk1, which recruits HAUS6 to spindle MTs and NEDD1 to the spindle and centrosomes during mitosis [6]. HAUS6 may therefore be a promising novel target for anticancer treatments. Further study should be done to investigate the mechanism by which HAUS6 knockdown activates the p53 pathway.

\section{Conclusions}

In summary, we show that HAUS6 upregulation is associated with shorter survival of CRC patients and increased cell viability in cultured CRC cells, while HAUS6 knockdown inhibits tumor growth and enhances 5-FU treatment by activating the p53/p21 pathway. HAUS6 may be a useful prognostic marker and chemotherapeutic target in CRC.

\section{Abbreviations}

CRC: colorectal cancer; qRT-PCR: quantitative reverse transcription - polymerase chain reaction; 5-FU: 5fluorouracil; MT: microtubule; NEDD1: neural precursor cell-expressed developmentally down-regulated protein 1; FJTCM: Fujian University of Traditional Chinese Medicine; $\mathrm{p}$-TNM: pathologic tumor-nodemetastasis; TMA: tissue microarray; EGFP: enhanced green fluorescent protein; MOI: multiplicity of infection; FACS: fluorescence activated cell sorting; SPF: specific pathogen-free; DEGs: differentially expressed genes.

\section{Declarations}

\section{Ethics approval and consent to participate}

Collection of the clinic samples and related experiments were approved by the Ethics Committee of FJTCM. Written, informed consent was obtained from all patients or their families. All animal maintenance and procedures were performed in strict accordance with the "Guide for the Care and Use of Laboratory Animals" and the "Principles for the Utilization and Care of Vertebrate Animals" and approved by the Animal Committee of FJTCM.

\section{Consent for publication}

All authors have agreed to publish this manuscript.

\section{Competing interests}


The authors have declared that no competing interest exists.

\section{Availability of data and materials}

The datasets used and/or analyzed during the current study are available from the corresponding author on reasonable request.

\section{Funding}

This study was supported by the National Natural Science Foundation of China $(81673721,81803882$, 81703913), the International Cooperative Project of Fujian Department of Science and Technology (2017I0007), and Natural Science Foundation of Fujian Province (2017J01846).

\section{Author contributions}

JP conceived and designed the experiments. AS, YLL and QZ conducted bioinformatatic analyses YLL, YQC and QZ provided the reagents. AS, LL and JC determined HAUS6 expression in CRC samples. AS, YC, $\mathrm{YH}, \mathrm{ZS}, \mathrm{XW}$ and $\mathrm{YQC}$ performed biological function analyses in vitro. $\mathrm{AS}, \mathrm{MW}, \mathrm{XL}, \mathrm{JC}$ and $\mathrm{LW}$ performed animal experiment. AS, $\mathrm{YH}$ and $\mathrm{MW}$ conducted rescued experiment. $\mathrm{AS}, \mathrm{YH}, \mathrm{ZS}, \mathrm{MW}$ and $\mathrm{XC}$ explored the underlying molecule mechanism. LL, YC, JC and YLL conducted data analysis. AS, YQC, JP, TJS wrote and revised the manuscript.

\section{Acknowledgments}

We thank Drs. Wei Lin, Weidong Zhu, Baochang He, Kehong Zhang and Guoqing Ji for helpful advice and discussions.

\section{References}

1. Nakayama G, Tanaka C, Kodera Y. Current Options for the Diagnosis, Staging and Therapeutic Management of Colorectal Cancer. Gastrointest Tumors. 2013;1:25-32.

2. Fakih MG. Metastatic colorectal cancer: current state and future directions. JClin Oncol. 2015;33:1809-24.

3. Del Vescovo V, Denti MA. microRNA and Lung Cancer. Adv Exp Med Biol. 2015;889:153-77.

4. Shen A, Chen Y, Liu L, Huang Y, Chen H, Qi F, et al. EBF1-Mediated Upregulation of Ribosome Assembly Factor PNO1 Contributes to Cancer Progression by Negatively Regulating the p53 Signaling Pathway. Cancer Res. 2019;79:2257-70.

5. Goshima G, Mayer M, Zhang N, Stuurman N, Vale RD. Augmin: a protein complex required for centrosome-independent microtubule generation within the spindle. J Cell Biol. 2008;181:421-9.

6. Zhu H, Coppinger JA, Jang CY, Yates JR 3rd, Fang G. FAM29A promotes microtubule amplification via recruitment of the NEDD1-gamma-tubulin complex to the mitotic spindle. J Cell Biol. 2008;183:835-48. 
7. Du L, Xu J, Li X, Ma N, Liu Y, Peng J, et al. Rumba and Haus3 are essential factors for the maintenance of hematopoietic stem/progenitor cells during zebrafish hematopoiesis. Development. 2011;138:619-29.

8. Hayward D, Metz J, Pellacani C, Wakefield JG. Synergy between multiple microtubule-generating pathways confers robustness to centrosome-driven mitotic spindle formation. Dev Cell. 2014;28:8193.

9. Lawo S, Bashkurov M, Mullin M, Ferreria MG, Kittler R, Habermann B, et al. HAUS, the 8-subunit human Augmin complex, regulates centrosome and spindle integrity. Curr Biol. 2009;19:816-26.

10. Nakaoka Y, Miki T, Fujioka R, Uehara R, Tomioka A, Obuse C, et al. An inducible RNA interference system in Physcomitrella patens reveals a dominant role of augmin in phragmoplast microtubule generation. Plant Cell. 2012;24:1478-93.

11. Goshima G, Scholey JM. Control of mitotic spindle length. Annu Ver Cell DevBiol. 2010;26:21-57.

12. Sánchez-Huertas C, Freixo F, Viais R, Lacasa C, Soriano E, Lüders J. Non-centrosomal nucleation mediated by augmin organizes microtubules in post-mitotic neurons and controls axonal microtubule polarity. Nat Commun. 2016;7:12187.

13. Kline-Smith SL, Walczak CE. Mitotic spindle assembly and chromosome segregation: refocusing on microtubule dynamics. Mol Cell. 2004;15:317-27.

14. Meunier S, Vernos I. Microtubule assembly during mitosis - from distinct origins to distinct functions? J Cell Sci. 2012;125:2805-14.

15. Chouinard G, Clément I, Lafontaine J, Rodier F, Schmitt E. Cell cycle-dependent localization of CHK2 at centrosomes during mitosis. Cell Div. 2013;8:7.

16. Holland AJ, Cleveland DW. Losing balance: the origin and impact of aneuploidy in cancer. EMBO Rep. 2012;13:501-14.

17. Wang Z, Fan M, Candas D, Zhang TQ, Qin L, Eldridge A, Wachsmann-Hogiu S, Ahmed KM, Chromy BA, Nantajit D, Duru N, He F, Chen M, Finkel T, Weinstein LS, Li JJ. Cyclin B1/Cdk1 coordinates mitochondrial respiration for cell-cycle G2/M progression. Dev Cell. 2014;29:217-32.

18. Jang SH, Kim AR, Park NH, Park JW, Han IS. DRG2 Regulates G2/M Progression via the Cyclin B1Cdk1 Complex. Mol Cells. 2016;39:699-704.

19. Sherr CJ. Cancer cell cycles. Science. 1996;274:1672-7.

20. Yuba-Kubo A, Kubo A, Hata M, Tsukita S. Gene knockout analysis of two gamma-tubulin isoforms in mice. Dev Biol. 2005;282:361-73.

21. Huang S, Tang R, Poon RY. BCL-W is a regulator of microtubule inhibitor-induced mitotic cell death. Oncotarget. 2016;7:38718-30.

22. Karna P, Rida PC, Pannu V, Gupta KK, Dalton WB, Joshi H, et al. A novel microtubule-modulating noscapinoid triggers apoptosis by inducing spindle multipolarity via centrosome amplification and declustering. Cell Death Differ. 2011;18:632-44. 
23. Paier CRK, Maranhão SS, Carneiro TR, Lima LM, Rocha DD, Santos RDS, Farias KM, Moraes-Filho MO, Pessoa C. Natural products as new antimitotic compounds for anticancer drug development. Clinics (Sao Paulo). 2018; 73: e813s.

24. Bates D, Eastman A. Microtubule destabilising agents: far more than just antimitotic anticancer drugs. Br J Clin Pharmacol. 2017;83:255-68.

25. Kollareddy M, Sherrard A, Park JH, Szemes M, Gallacher K, Melegh Z, et al. The small molecule inhibitor YK-4-279 disrupts mitotic progression of neuroblastoma cells, overcomes drug resistance and synergizes with inhibitors of mitosis. Cancer Lett. 2017;403:74-85.

26. Taylor BF, McNeely SC, Miller HL, States JC. Arsenite-induced mitotic death involves stress response and is independent of tubulin polymerization. Toxicol Appl Pharmacol. 2008;230:235-46.

27. Ehrhardt H, Pannert L, Pfeiffer S, Wachter F, Amtmann E, Jeremias I. Enhanced anti-tumour effects of Vinca alkaloids given separately from cytostatic therapies. Br J Pharmacol. 2013;168:1558-69.

28. Po'uha ST, Kavallaris M. Gamma-actin is involved in regulating centrosome function and mitotic progression in cancer cells. Cell Cycle. 2015;14:3908-19.

29. Dimitri Lodygin A, Menssen H, Hermeking. Induction of the Cdk inhibitor p21 by LY83583 inhibits tumor cell proliferation in a p53-independent manner. J Clin Invest. 2002;110:1717-27.

30. Wang Q, He G, Hou M, Chen L, Chen S, Xu A, et al. Cell Cycle Regulation by Alternative Polyadenylation of CCND1. Sci Rep. 2018;8:6824.

31. Li M, Ke J, Wang Q, Qian H, Yang L, Zhang X, et al. Upregulation of ROCK2 in gastric cancer cell promotes tumor cell proliferation, metastasis and invasion. Clin Exp Med. 2017;17:519-29.

32. Gao X, Dai M, Li Q, Wang Z, Lu Y, Song Z. HMGA2 regulates lung cancer proliferation and metastasis. Thorac Cancer. 2017;8:501-10.

33. Harper JW, Adami GR, Wei N, Keyomarsi K, Elledge SJ. The p21 Cdk-interacting protein Cip1 is a potent inhibitor of G1 cyclin-dependent kinases. Cell. 1993;75:805-16.

34. Jung YS, Qian Y, Chen X. Examination of the expanding pathways for the regulation of $p 21$ expression and activity. Cell Signal. 2010;22:1003-12.

35. el-Deiry WS, Tokino T, Velculescu VE, Levy DB, Parsons R, Trent JM, et al. WAF1, a potential mediator of p53 tumor suppression. Cell. 1993;75:817-25.

36. Vogelstein B, Lane D, Levine AJ. Surfing the p53 network. Nature. 2000;408:307-10.

\section{Figures}


A

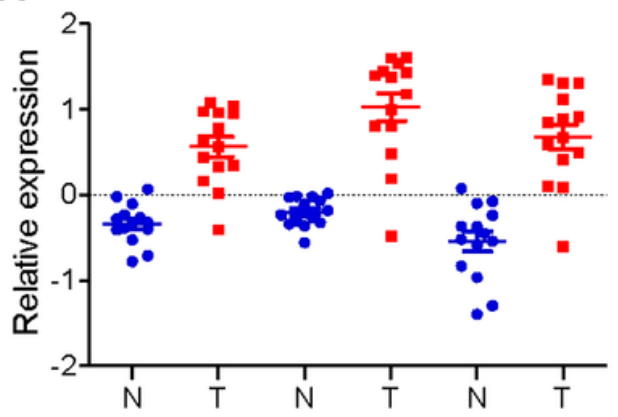

D

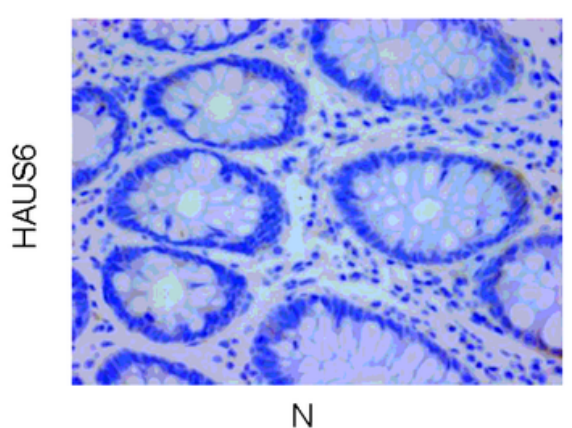

E

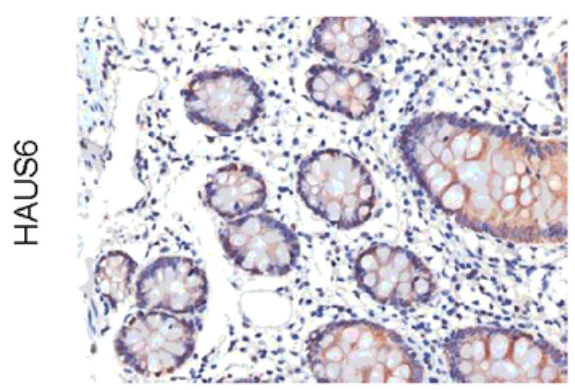

$\mathrm{N}$
B
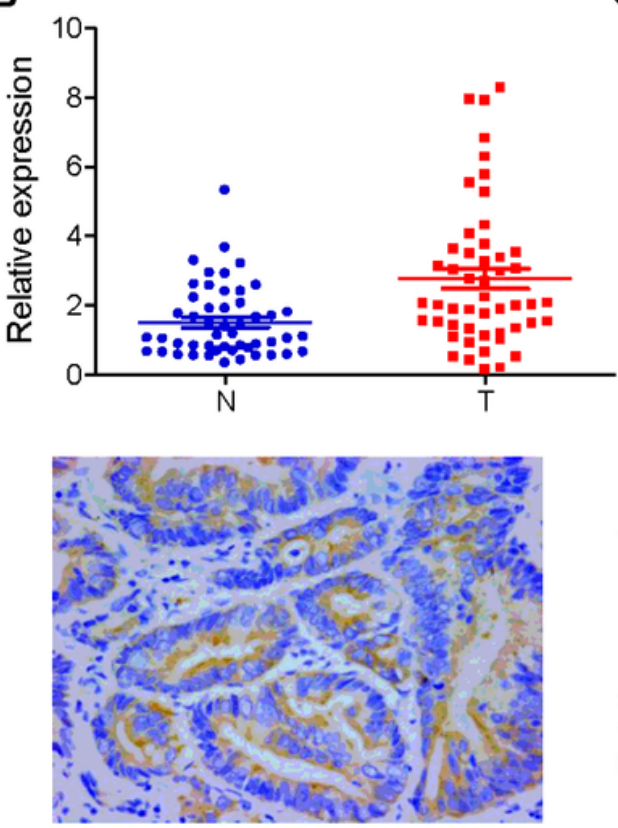

$\mathrm{T}$

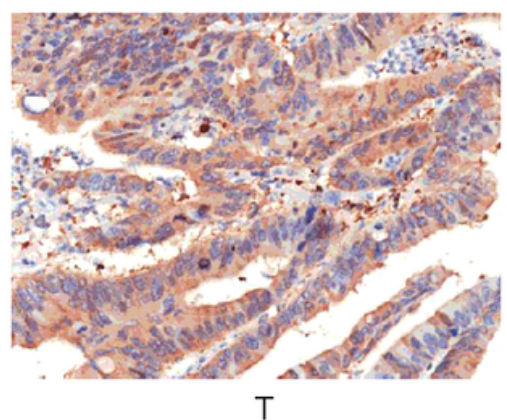

C
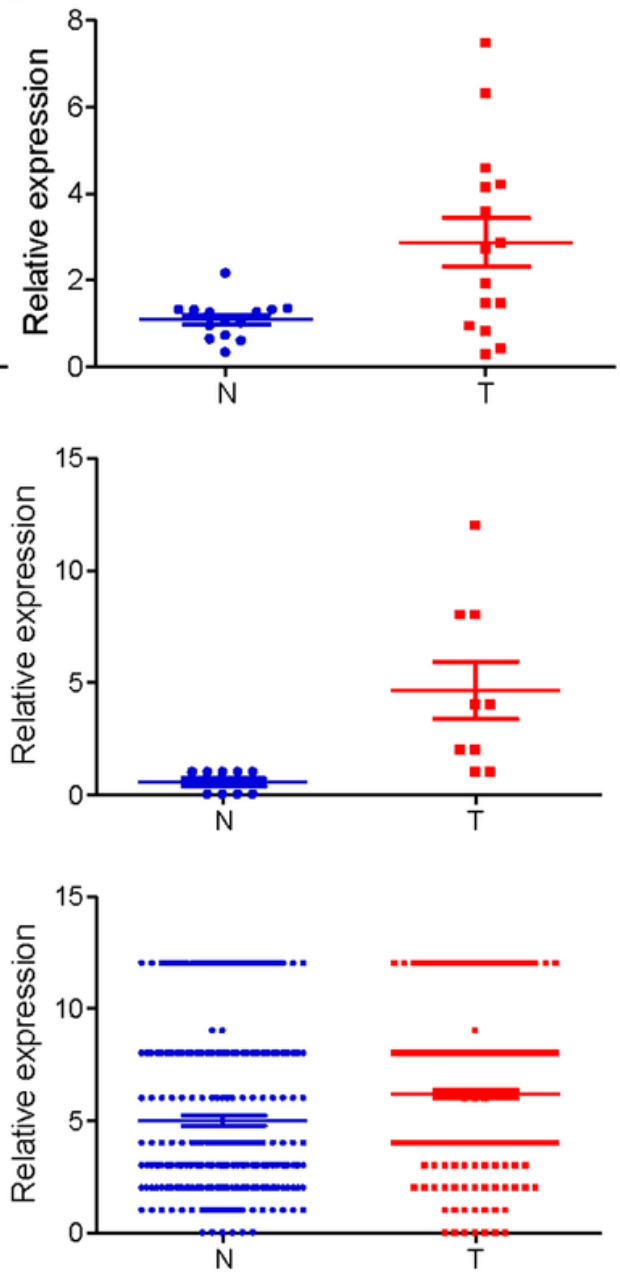

Figure 1

HAUS6 mRNA and protein are up-regulated in CRC tissues. (A) HAUS6 expression (3 different probes) in 14 matched $\mathrm{CRC}$ and noncancerous colorectal tissues from our previously described gene expression profile microarrays (GEO Submission: GSE113513). Each dot represents 1 tissue; Mean $\pm S D ; * p<0.05$, vs. $\mathrm{N}$, paired Student's t test. (B-C) Quantitative RT-PCR determination of HAUS6 mRNA expression in 36 matched CRC and noncancerous colorectal tissues collected by our laboratory and 15 matched CRC and noncancerous colorectal tissues from commercially available cDNA arrays. GAPDH was used as an internal control. Mean $\pm S D$; * $p<0.05$, vs. $N$, by paired Student's t test. (D-E) Immunohistochemistry to determine HAUS6 protein levels in 10 matched CRC and noncancerous colorectal tissues collected by our laboratory (Magnification: 40x), and 260 matched CRC and noncancerous colorectal tissues from commercially available tissue microarrays (Magnification: 20x). Representative images are shown on the lower panel. Mean $\pm S D ;{ }^{*} p<0.05$, vs. N, by paired Student's t test. T: colorectal cancer tissues; N: noncancerous colorectal tissues. 
A

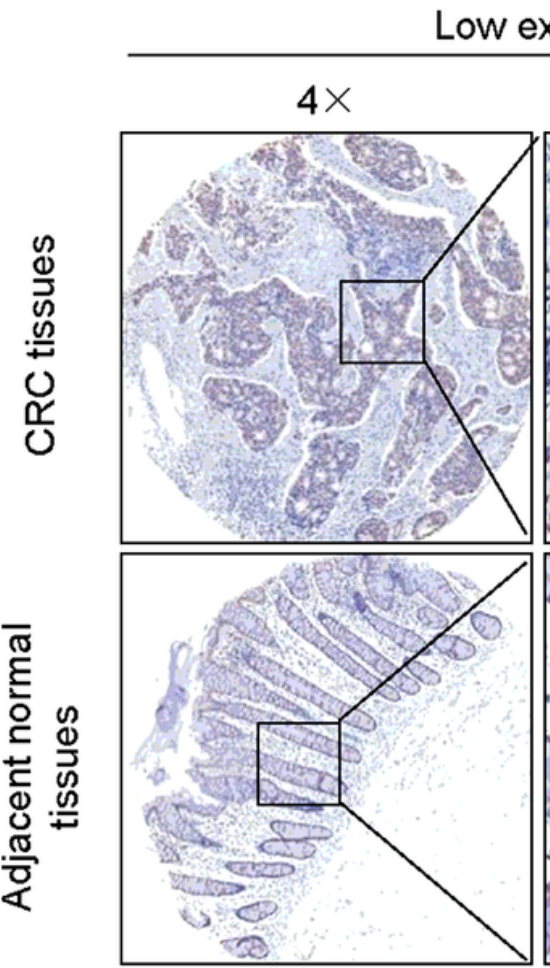

Low expression

High expression
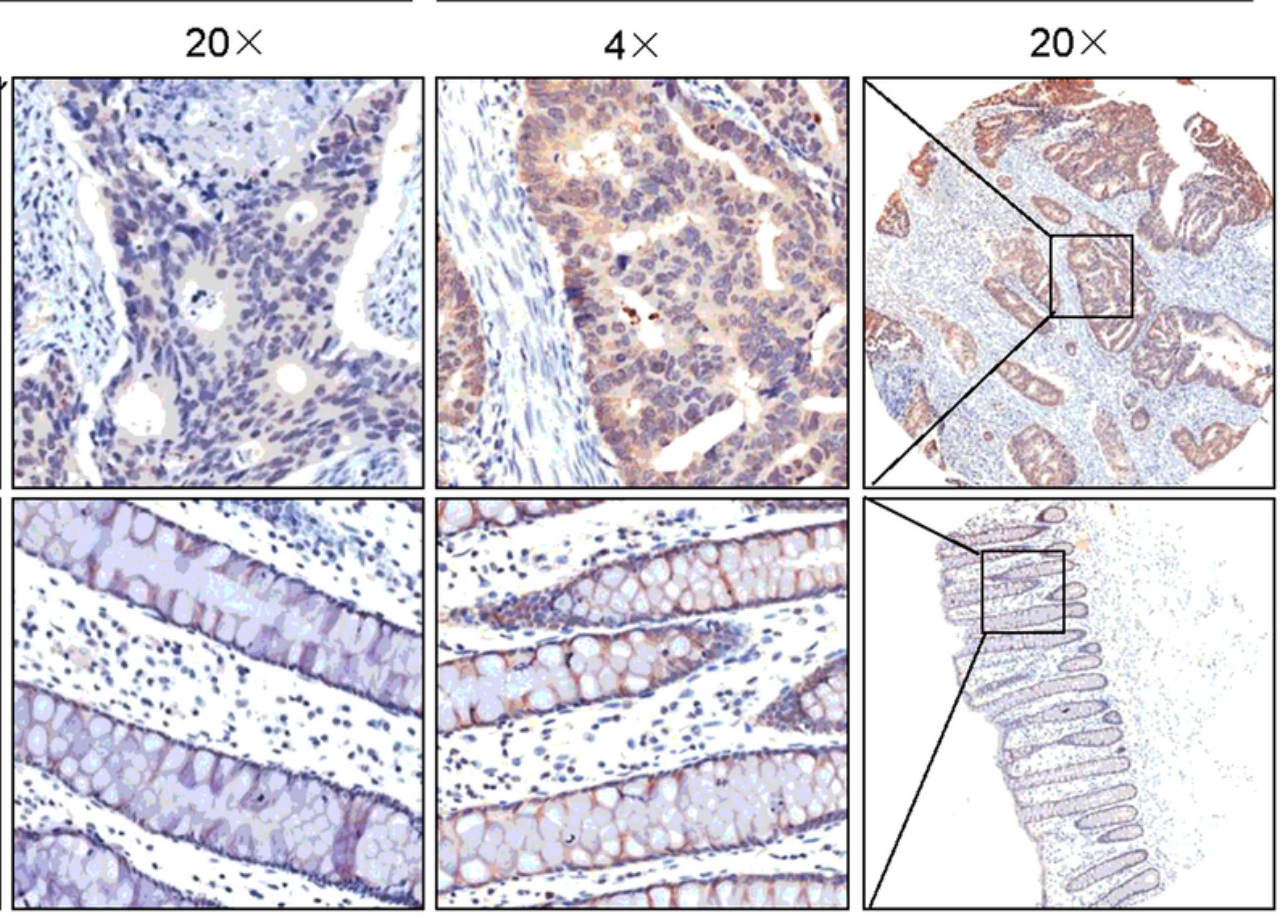

B

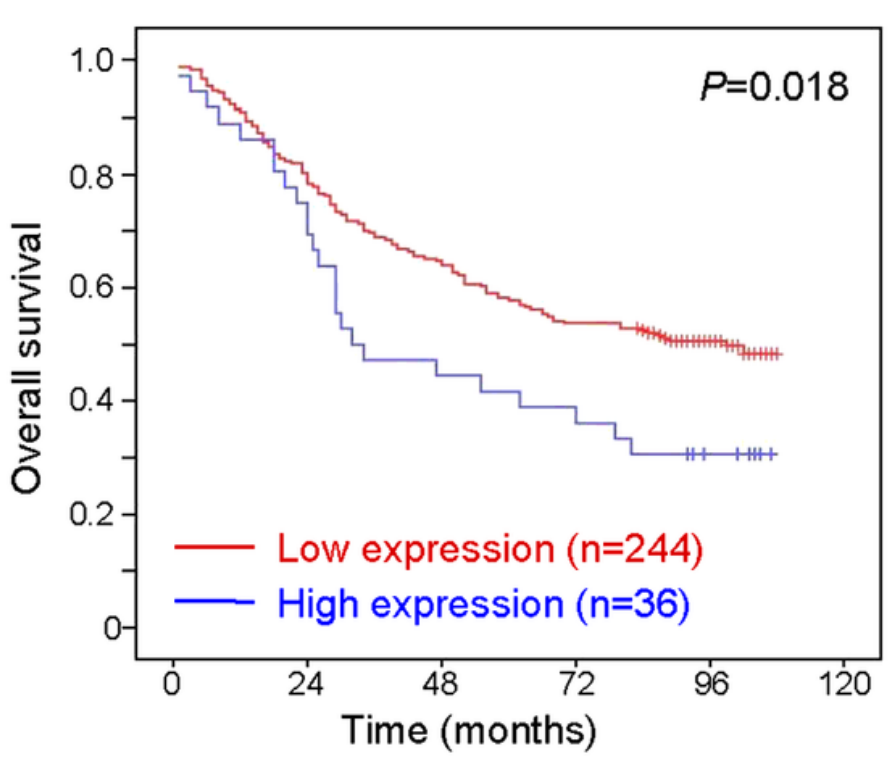

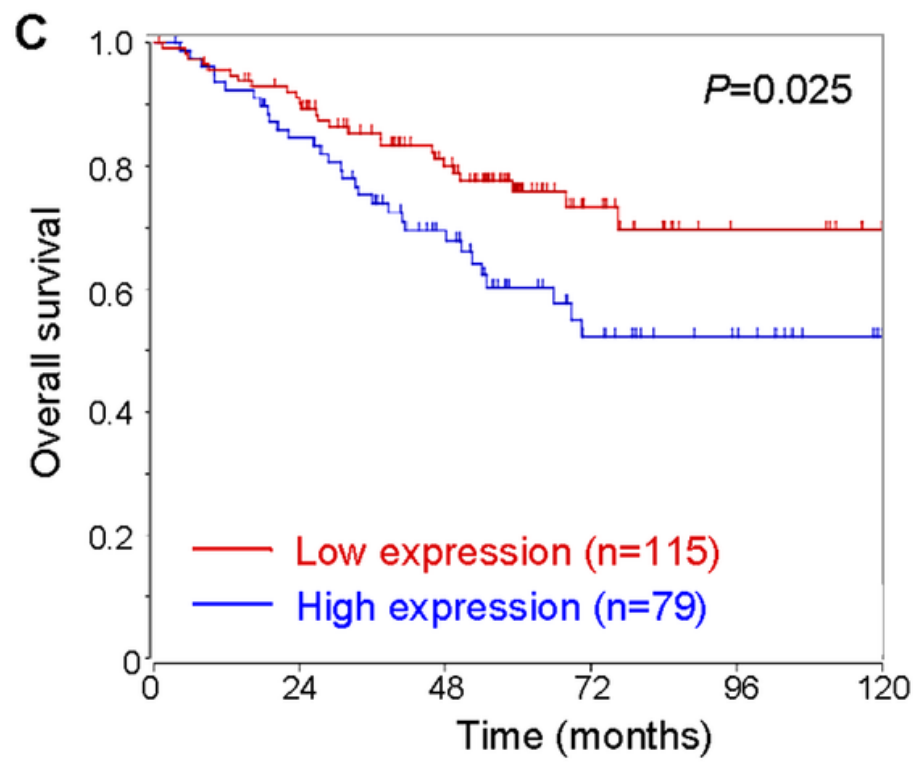

Figure 2

High HAUS6 expression is associated with shorter overall survival in CRC patients. (A) Overall survival of 194 CRC patients with low and high HAUS6 mRNA expression (GEO Submission: GSE24551), based on data in the public microarray dataset that were analyzed through the R2 bioinformatic platform by Kaplan-Meier using the log-rank test. (B) Kaplan-Meier plots showing overall survival of 280 CRC patients with low and high HAUS6 protein expression by Kaplan-Meier using the log-rank test. (C) Representative immunohistochemistry images showing high or low HAUS6 expression. Magnification: $4 \times$ and 20x. 
A

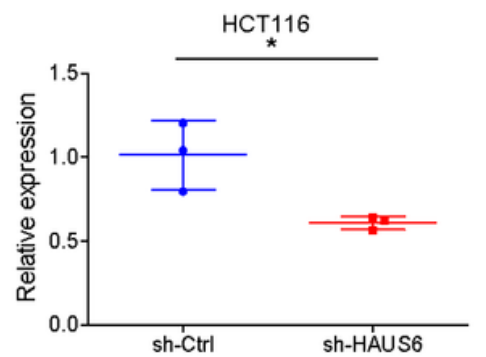

B

C
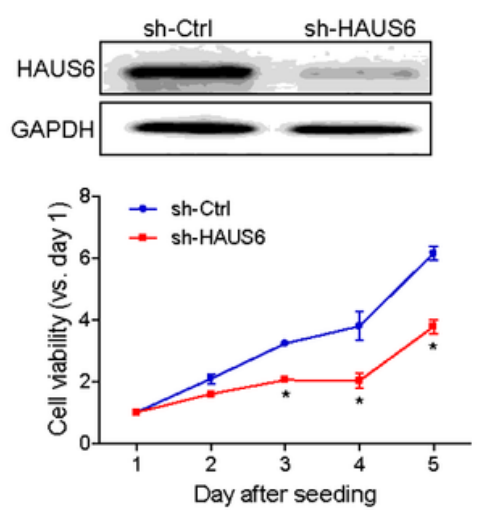
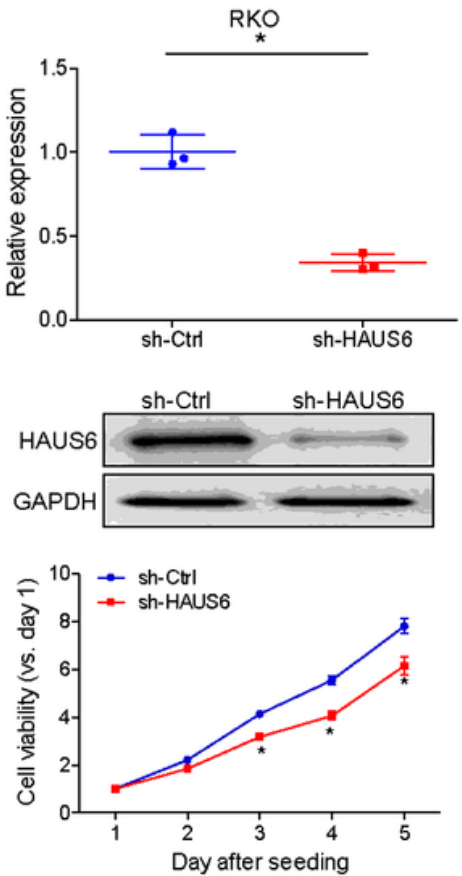
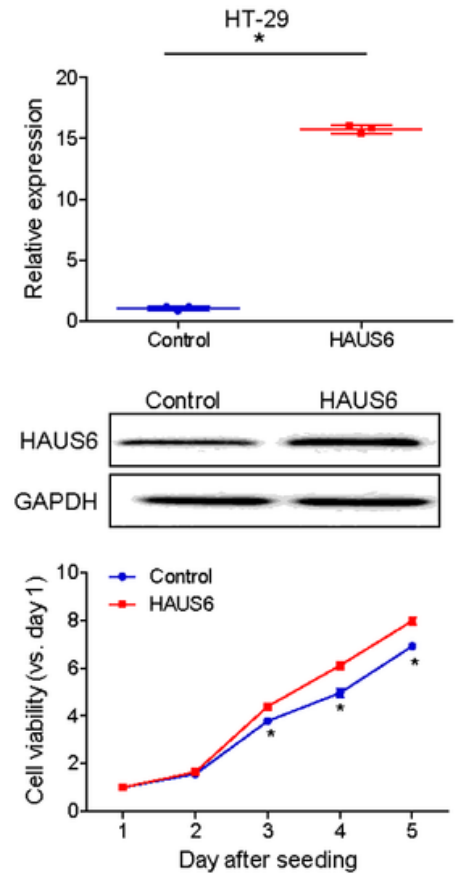

D HCT116
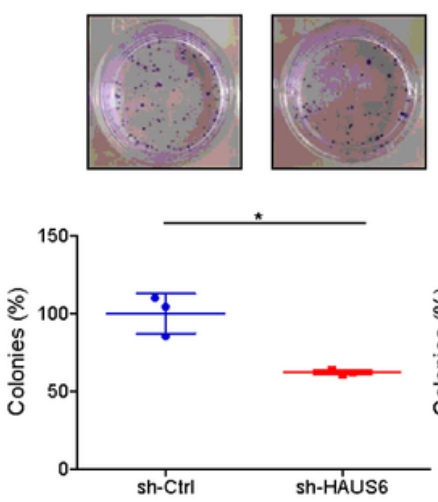

RKO
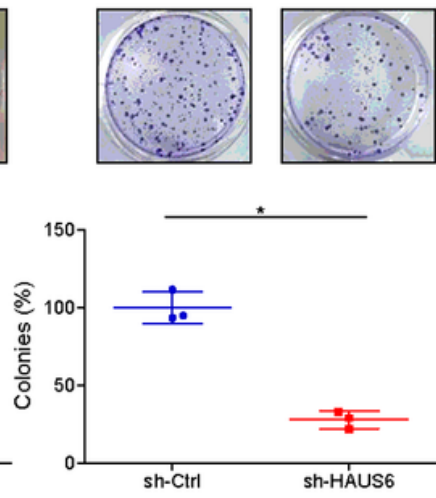

E
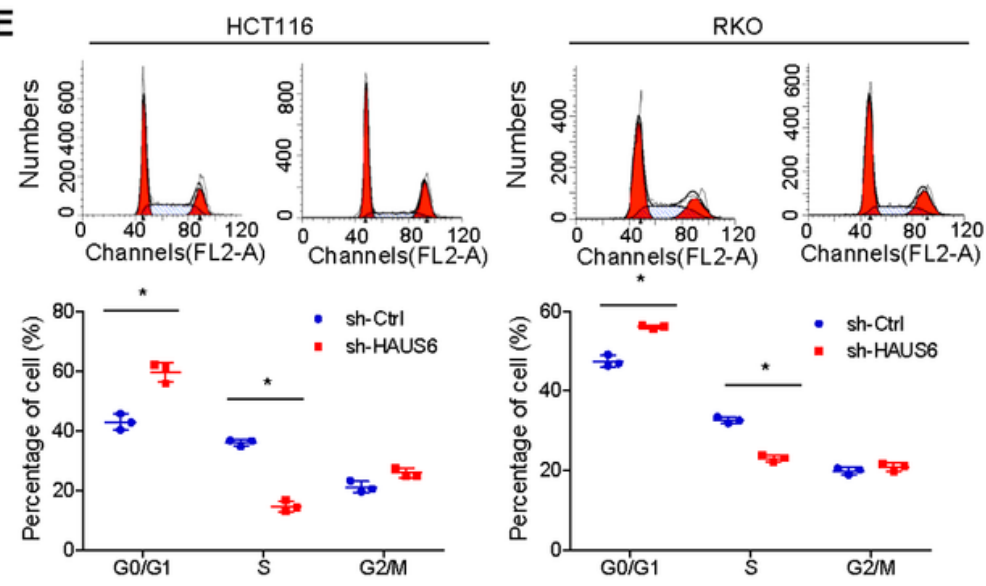

Figure 3

HAUS6 knockdown reduces CRC cell growth and proliferation in vitro. (A-B) The mRNA and protein levels of HAUS6 in HCT116 (left panel) and RKO (middle panel) cells after knockdown of HAUS6, and HT-29 (right panel) cells after over-expression HAUS6 were assessed by quantitative RT-PCR and western blotting $(n=3)$. GAPDH was used as a loading control. For HAUS6 mRNA expression, Mean $\pm S D ; * p<0.05$, vs. sh-Ctrl or Control, by independent Student's t test. (C) Viability of HCT116 (left panel) and RKO (middle panel) cells after knockdown of HAUS6, and HT-29 (right panel) cells after over-expression HAUS6 was determined by CCK-8 assay. Data are normalized to viability on day 1 and are represented as fold changes. Mean $\pm S D ; n=6$; * $p<0.05$, vs. sh-Ctrl or Control, by independent Student's t test. (D) Survival of HCT116 (left panel) and RKO (right panel) cells transduced with shRNA against HAUS6 or a control shRNA was assessed in the colony formation assay. Representative images of colonies are shown above the graphs. Cell survival was normalized to the sh-Ctrl group. Mean $\pm S D ; n=3 ; * p<0.05$, vs. sh-Ctrl, by independent Student's t test. (E) Percentage of HCT116 (left panel) and RKO (right panel) cells 
transduced with shRNA against HAUS6 or a control shRNA in G0/G1, S, and G2/M phases was assessed by flow cytometry. Representative flow cytometry plots are shown above, and the percentages of cells in each phase are shown below. Mean $\pm S D ; n=3 ; * p<0.05$, vs. sh-Ctrl, by independent Student's t test.

A

B
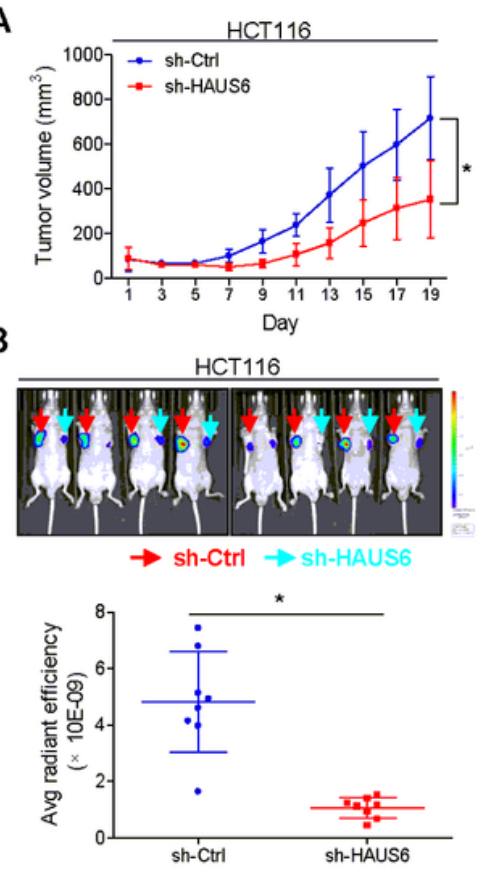

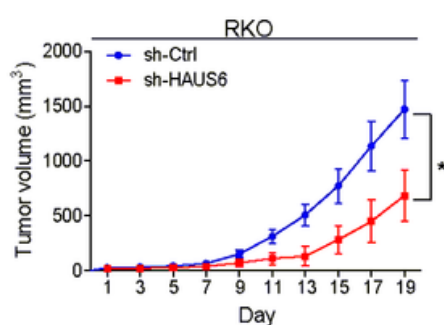

RKO

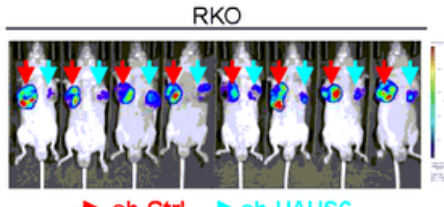

$\rightarrow$ sh-Ctrl $\rightarrow$ sh-HAUS6

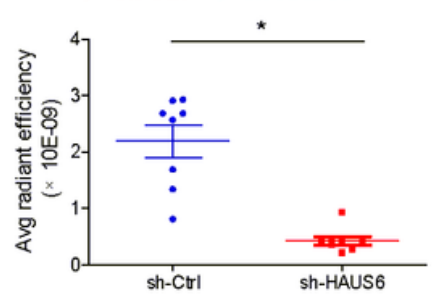

C

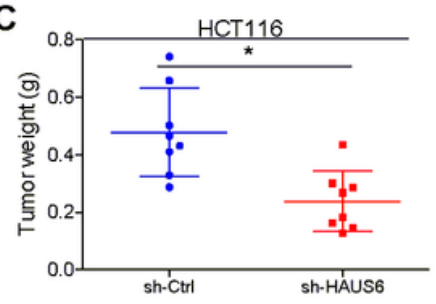

D
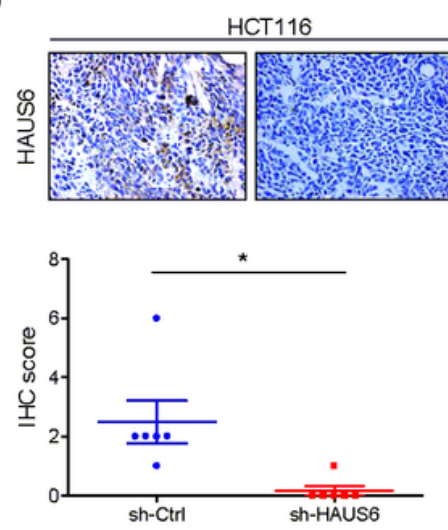
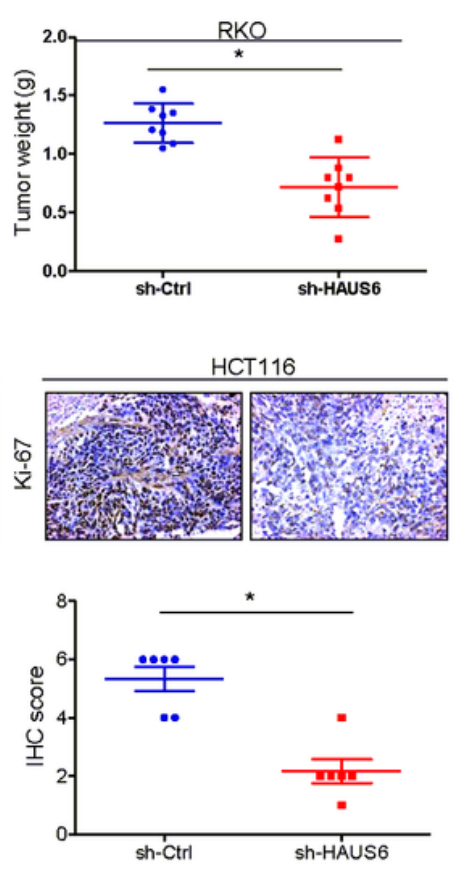

Figure 4

HAUS6 knockdown reduces tumor growth and cell proliferation in vivo. (A) Tumor volume in BALB/c nude mice injected with HCT116 (left panel) or RKO (right panel) cells transduced with shRNA against HAUS6 or control shRNA. Mean $\pm S D ; n=8$; * $p<0.05$, vs. sh-Ctrl, by independent Student's t test. (B) GFP fluorescence in BALB/c nude mice injected with HCT116 (left panel) or RKO (right panel) cells transduced with a GFP reporter and shRNA against HAUS6 or control shRNA. Representative fluorescence images are shown above, and quantitation of GFP intensity is shown below. Mean $\pm S D ; n=8$; * $p<0.05$, vs. sh-Ctrl, by independent Student's t test. (C) Tumor weight in BALB/c nude mice injected with HCT116 (left panel) or RKO (left panel) cells transduced with shRNA against HAUS6 or control shRNA on day 26 day after injection. Mean $\pm S D ; n=8 ; *$ p $<0.05$, vs. sh-Ctrl, by independent Student's $t$ test. (D) HAUS6 and Ki-67 protein levels in excised tumors from BALB/c nude mice injected with HCT116 cells transduced with shRNA against HAUS6 or control shRNA. Representative immunohistochemistry images are shown above. Magnification: 40x; Mean $\pm S D ; n=6$; * $p<0.05$, vs. sh-Ctrl, by independent Student's t test. 
A

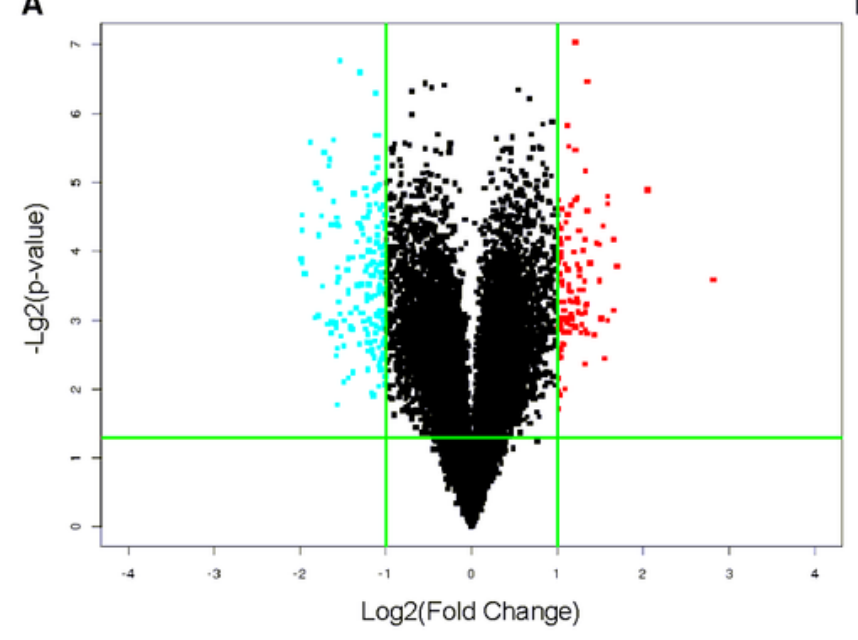

and

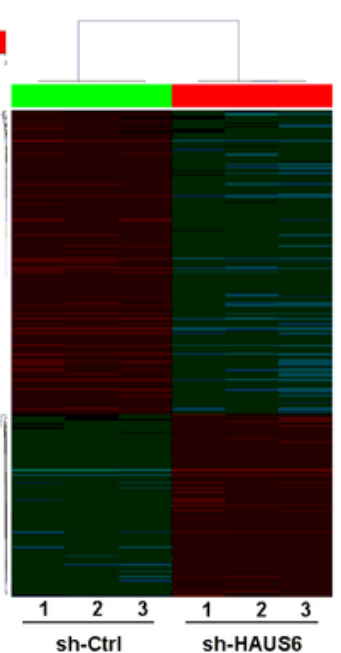

F

E
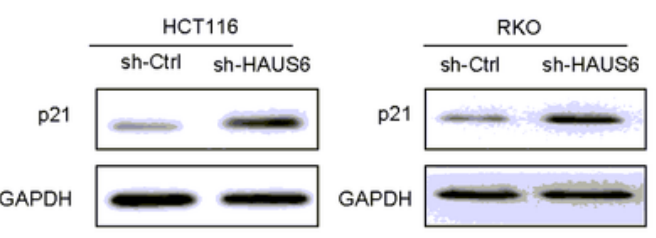

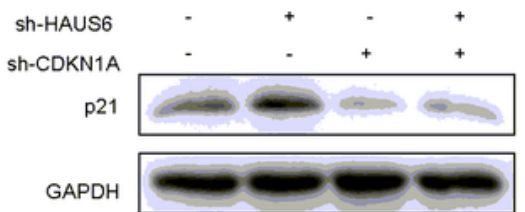

C

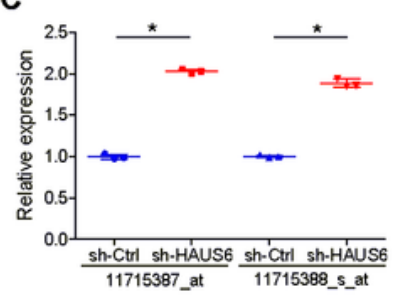

D

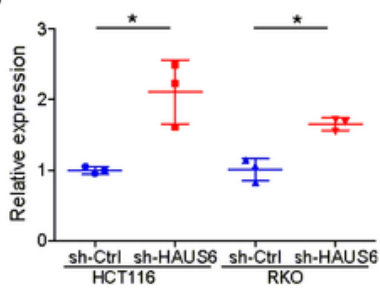

G $\rightarrow$ sh-Ctrl $\rightarrow$ sh-CDKN1A

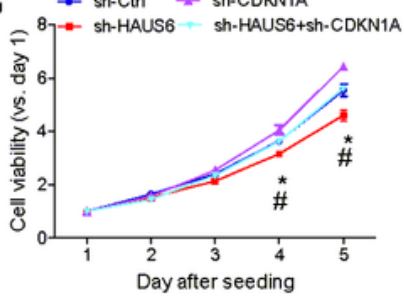

H

I

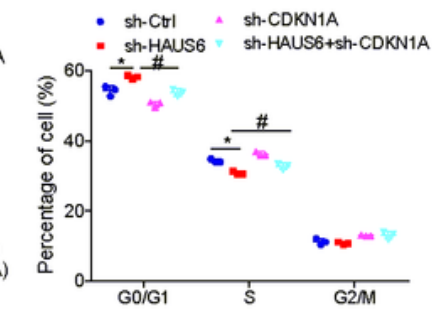

J

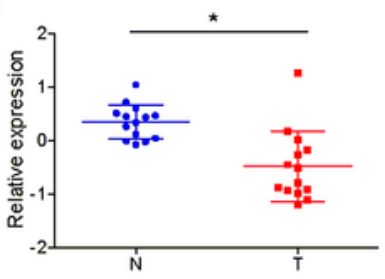

K
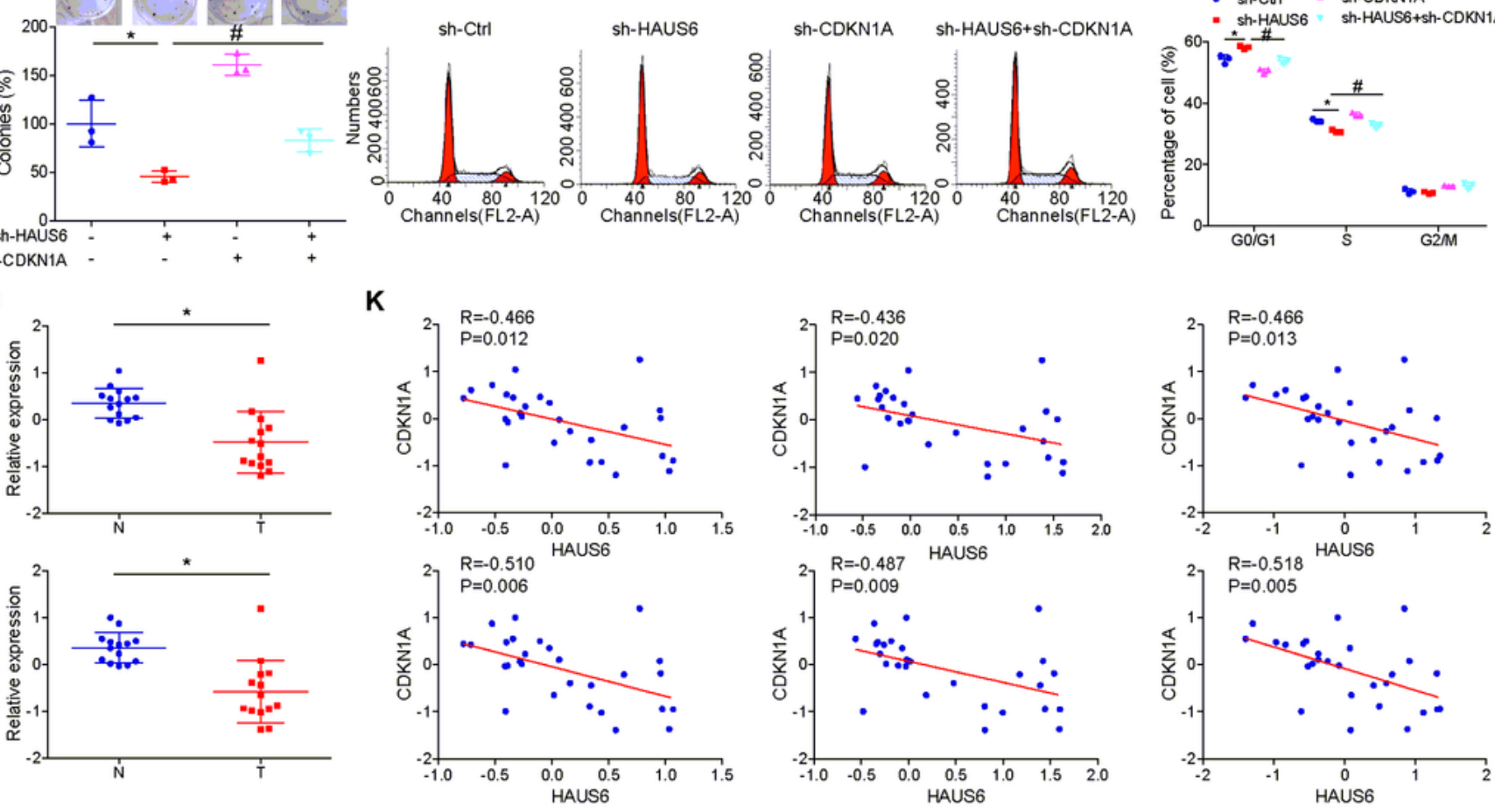

Figure 5

HAUS6 knockdown suppresses cell proliferation by increasing p21 expression. (A-B) A volcano plot and hierarchical clustering plot showing DEGs (fold change $>2, p<0.05$ ) between HCT116 cells transduced with shRNA against HAUS6 or control shRNA (n=3). (C-D) CDKN1A/p21 mRNA levels was assessed by cDNA microarray in HCT116 cells transduced with shRNA against HAUS6 or control shRNA and quantitative RT-PCR in HCT116 and RKO cells transduced with shRNA against HAUS6 or control shRNA. 
GAPDH was used as an internal control. Mean $\pm S D ; n=3 ;$ * $p<0.05$, vs. sh-Ctrl by independent Student's $t$ test. (E) Protein levels of p21 in HCT116 and RKO cells after HAUS6 knockdown was assessed by western blot $(n=3)$. GAPDH was used as a loading control. ( $F)$ Expression of p21 protein in HCT116 cells transduced with shRNAs against HAUS6, CDKN1A or both, or control shRNA was assessed by western blot $(n=3)$. GAPDH was used as a loading control. (G) Viability of HCT116 cells transduced with shRNA against HAUS6, CDKN1A, or both, or control shRNA. Data were normalized to day 1 and presented as fold changes. Mean $\pm S D ; n=6 ;{ }^{*} p<0.05$, vs. sh-Ctrl and \# $p<0.05$, vs. sh-HAUS6, by one-way ANOVA with LSD post hoc test. $(\mathrm{H})$ Cell survival was assessed by colony formation assay. Representative images of colonies (upper panel) and numbers of colonies (lower panel) are shown. The number of colonies was normalized to the sh-Ctrl group. Mean $\pm S D ; n=3$; * $p<0.05$, vs. sh-Ctrl and $\# p<0.05$, vs. sh-HAUS6, by oneway ANOVA with LSD post hoc test. (I) Percentages of HCT116 cells transduced with shRNA against HAUS6, CDKN1A, or both, or control shRNA that were in G0/G1, S, or G2/M phase was determined by flow cytometry. Representative flow cytometry plots are shown on the left. Mean $\pm S D ; n=3 ; * p<0.05$, vs. sh-Ctrl by independent Student's $t$ test* $p<0.05$, vs. sh-Ctrl and $\# p<0.05$, vs. sh-HAUS6, by one-way ANOVA with LSD post hoc test. (J) CDKN1A mRNA levels in 14 matched pairs of CRC and non-cancerous tissues was assessed by two microarray probes $(n=14)$. (K) Pearson's rank correlation analysis between HAUS6 expression (three microarray probes) and CDKN1A expression (two microarray probes) in CRC tissues. 
A
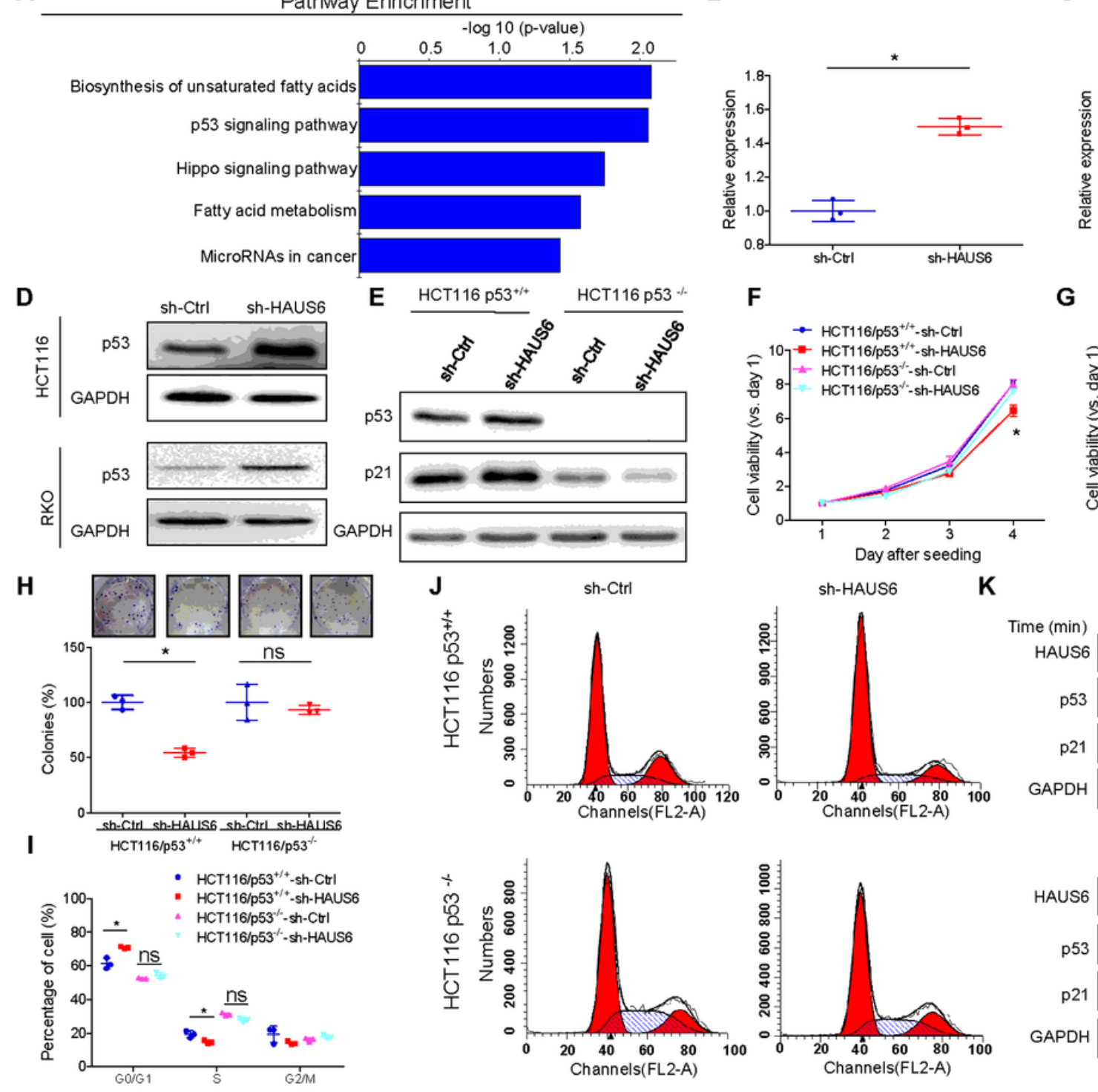

C

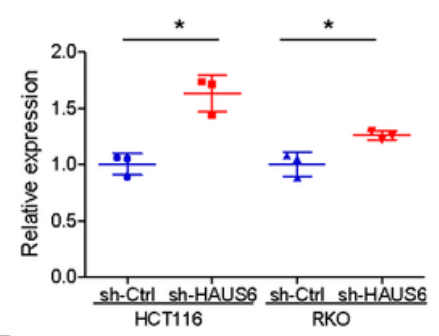

G

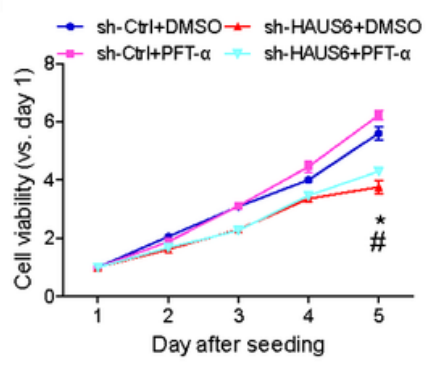

sh-Ctrl+CHX

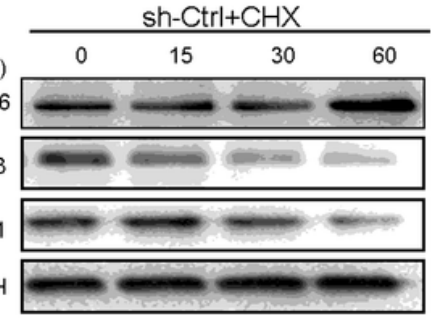

sh-HAUS6+CHX

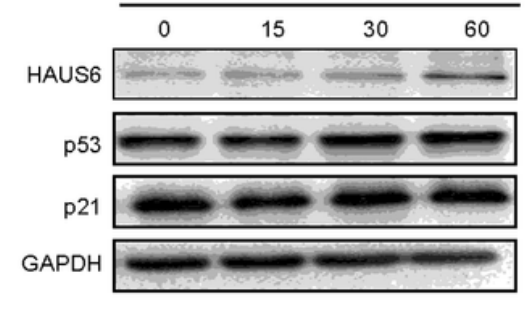

Figure 6

HAUS6 knockdown inhibits cell proliferation by activating the p53/p21 pathway. (A) The top five pathways enriched in DEGs was identified by KEGG pathway enrichment analysis (all p<0.05). (B-C) TP53 mRNA levels in cells transduced with shRNA against HAUS6 or control shRNA was assessed by microarray (HCT116 cells) and quantitative RT-PCR (HCT116 and RKO cells). Mean $\pm S D ; n=3$; * $p<0.05$, vs. sh-Ctrl, by independent Student's t test. GAPDH was used as an internal control. (D) Protein levels of p53 in HCT116 and RKO cells after HAUS6 knockdown was detected by western blot $(n=3)$. GAPDH was used as a loading control. (E) Protein levels of p53 in HCT116/p53+/+ and HCT116/p53-/- cells after HAUS6 knockdown was assessed by western blot $(n=3)$. GAPDH was used as a loading control. ( $F$ ) Viability of HCT 116/p53+/+ cells and HCT116/p53-/- cells transduced with shRNA against HAUS6 or control shRNA. Results were normalized to viability on day 1 . Mean $\pm S D ; n=6$; ${ }^{*} p<0.05$, vs. sh-Ctrl of HCT116/p53+/+ cells or HCT116/p53-/- cells, by independent Student's t test. (G) Cell viability in HCT116 cells treated with p53 inhibitor. Results were normalized to viability on day 1 . Mean $\pm S D ; n=6 ; * p<0.05$, vs. sh-Ctrl and $\#<0.05$, vs. sh-HAUS6+DMSO by one-way ANOVA with LSD post hoc test. (H) Survival of HCT116/p53+/+ cells 
and HCT116/p53-/- cells transduced with shRNA against HAUS6 or control shRNA. Representative images of colonies are shown in the upper panel. Cell survival was normalized relative to the sh-Ctrl group. Mean $\pm S D ; n=3$; * $p<0.05$, vs. sh-Ctrl of HCT116/p53+/+ cells, by independent Student's $t$ test. (I-J) Cell cycle distribution of HCT116/p53+/+ and HCT116/p53-/- cells transduced with shRNA against HAUS6 or control shRNA were determined by FACS analysis, (I) the representative flow cytometry plots were shown. (J) The percentages of cells in $\mathrm{G} 0 / \mathrm{G} 1, \mathrm{~S}$, and $\mathrm{G} 2 / \mathrm{M}$ phases were analyzed. Mean $\pm S D ; n=3$; Mean $\pm S D ; n=3 ;$ * $p<0.05$, vs. sh-Ctrl of HCT116/p53+/+ cells, by independent Student's t test. (K) HAUS6, p53 and p21 protein levels in RKO cells transduced with shRNA against HAUS6 or control shRNA after cycloheximide (CHX) treatment was determined by western blot $(n=3)$. GAPDH was used as a loading control. 

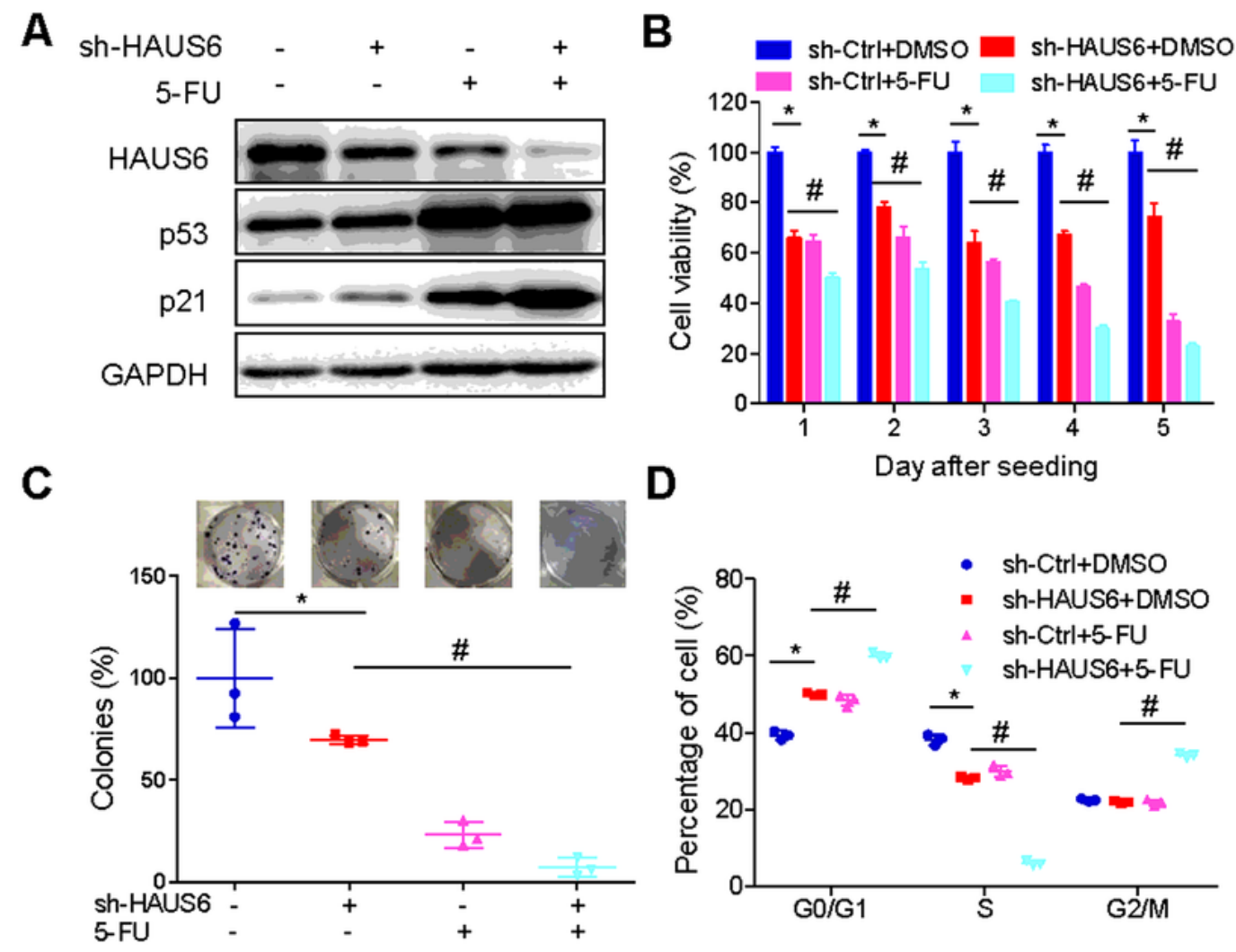

D Day after seeding

E
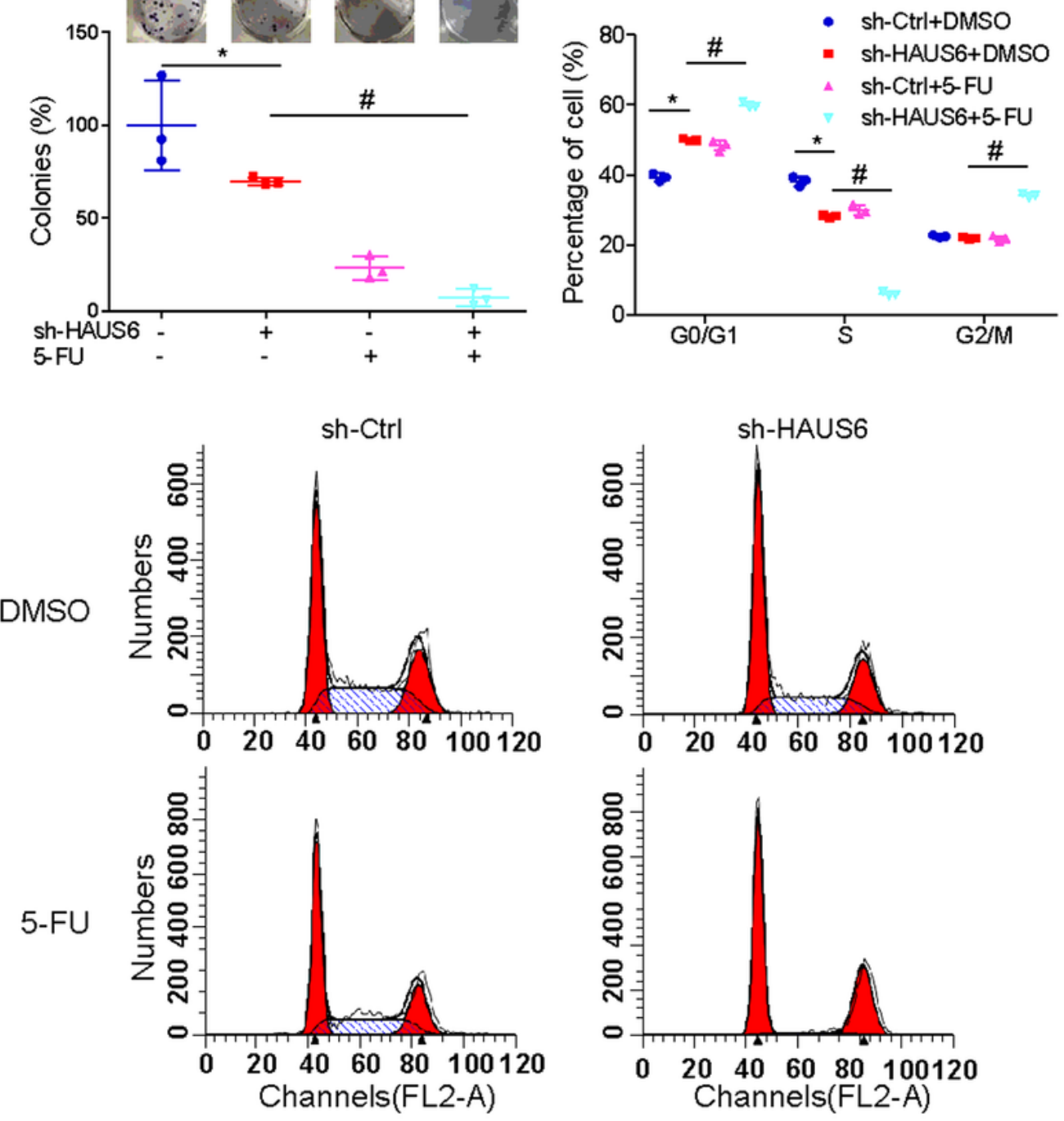

\section{Figure 7}

HAUS6 knockdown sensitizes CRC cells to 5-FU treatment by activating the p53/p21 pathway. (A) HAUS6, p53 and p21 protein levels in HCT116 cells transduced with shRNA against HAUS6 or control shRNA and treated with 5-FU, was determined by western blot $(n=3)$. GAPDH was used as a loading control. (B) Viability of HCT116 cells transduced with shRNA against HAUS6 or control shRNA and treated with 5-FU, was determined by CCK-8 assay. Results were normalized to the sh-Ctrl group. Mean $\pm S D ; n=6 ; * 0<0.05$, 
vs. sh-Ctrl, \# p<0.05 vs. sh-HAUS6+DMSO, by one-way ANOVA with LSD post hoc test. (C) Survival of HCT116 cells transduced with shRNA against HAUS6 or control shRNA and treated with 5-FU, was assessed by colony formation assay. Representative images of colonies are shown on the upper panel, and cell survival normalized to the sh-Ctrl group is shown on the lower panel. Mean $\pm S D ; n=3 ;{ }^{*} p<0.05$, vs. sh-Ctrl, \# $p<0.05$ vs. sh-HAUS6+DMSO, by one-way ANOVA with LSD post hoc test. (D-E) Cell cycle distribution of HCT116 cells transduced with shRNA against HAUS6 or control shRNA and treated with 5FU, was assessed by flow cytometry. (D)The representative flow cytometry plots are shown. (E) The percentages of cells in G0/G1, S, and G2/M phase are shown. Mean $\pm S D ; n=3$; * $p<0.05$, vs. sh-Ctrl, \# p $<0.05$ vs. sh-HAUS6+DMSO, by one-way ANOVA with LSD post hoc test.

\section{Supplementary Files}

This is a list of supplementary files associated with this preprint. Click to download.

- SupplementaryFigure1.tif

- SupplementaryFigure1.tif

- SupplementaryFigure2.tif

- SupplementaryFigure2.tif

- Supplementarytable.doc

- Supplementarytable.doc 